\title{
CYP75 gene family in barley
}

\author{
Vikhorev A.V. ${ }^{1,2 *}$, Gerasimova S.V. ${ }^{1}$, Strygina K.V. ${ }^{1,3}$, Khlestkina E.K. ${ }^{1,3}$ \\ ${ }^{1}$ Institute of Cytology and Genetics, SB RAS, Novosibirsk, Russia \\ ${ }^{2}$ Novosibirsk State University, Novosibirsk, Russia \\ ${ }^{3}$ N.I. Vavilov Research Institute of Plant Industry, St. Petersburg, Russia \\ *e-mail:vikhorev@bionet.nsc.ru
}

Key words: anthocyanin biosynthesis, CYP75, flavonoid pigments, gene evolution, Hordeum, P450

Motivation and Aim: Anthocyanins are secondary metabolites of plants playing multiple biological functions. These compounds could be accumulated in many plants, including barley (Hordeum vulgare L.). Important structural genes in the pathway of anthocyanin biosynthesis are flavonoid 3'-hydroxylase $\left(F 3^{\prime} H, C Y P 75 B\right)$ and flavonoid 3',5'-hydroxylase $\left(F 3^{\prime} 5^{\prime} H\right.$, CYP75A). These genes catalyze the hydroxylation of B-ring of anthocyanins and direct the pathway of biosynthesis towards the formation of magenta pigments cyanidins and blue pigments delphinidins, respectively [1]. However, these genes are poorly studied in barley. The aim of this study was to identify and characterize duplicated copies of the $F 3^{\prime} H$ and $F 3^{\prime} 5^{\prime} H$ genes in the barley genome.

Methods and Algorithms: Homologous sequences of $F 3^{\prime} H$ and $F 3^{\prime} 5^{\prime} H$ was found in IPK Barley BLAST Server database using BLASTN search. In silico analysis of the genes was carried out using Multalin, FGENESH+, New PLACE and InterPro software. The construction of the UPGMA tree, the calculation of $K a / K s$ ratio and calculation of divergence time was performed using MEGA v.606 software with 1000 bootstrap replicates to assess the branch support. Analysis of expression of genes was made using qPCR.

Results: In barley genome we identified one homologous copy belonging to the family CYP75B (chromosome 6HS) and three homologous copies belonging to the family CYP75A (chromosomes 6HL, 6HS and 7HS). All found genes have a cytochrome P450 domain, except for the pseudogenic copy designated $F 3^{\prime} 5^{\prime} H-3$ (6HS). It was shown that genes $F 3$ ' $H-1$ and $F 3$ ' $H-2$ are responsible for the accumulation of magenta pigments in the pericarp and stem, respectively. The gene $F 3^{\prime} 5^{\prime} \mathrm{H}-1$ is responsible for the accumulation of blue pigments in the aleurone layer of barley grain. Genes $F 3$ '5 ' $H-2$, F3'5' $H-3$ and $F 3^{\prime} 5^{\prime} H-4$ do not participate in the biosynthesis of anthocyanins in the studied tissues.

Conclusion: In current work one new $F 3$ ' $H$ and three $F 3$ ' 5 ' $H$ genes were described for the first time. The subfunctionalization of $F 3^{\prime} H-1, F 3^{\prime} H-2$ and $F 3^{\prime} 5^{\prime} H-1$ genes expressing in tissue-specific activity and pseudogenization of $F 3^{\prime} 5^{\prime} H-3$ gene were shown. The results obtained are important for understanding the features of anthocyanin biosynthesis in barley and could be used for the manipulation of the content of diverse anthocyanins in different parts of barley.

Acknowledgements: This study was partially by the RFBR (No. 18-416-543007).

\section{References}

1. Shoeva O.Y., Mock H.P., Kukoeva T.V., Börner A., Khlestkina E.K. Regulation of the flavonoid biosynthesis pathway genes in purple and black grains of Hordeum vulgare. PloS ONE. 2016; 11(10):e0163782. 